\title{
Magnetic field analysis of Primary Permanent Magnet Linear Motor Based on Halbach Distribution
}

\author{
Xiuping Wang ${ }^{*}$, Yan Li, Chuhan Yang, Chunyu Qu \\ School of Electrical Power, Shenyang Institute of Engineering, Shenyang, China
}

\begin{abstract}
The rail transit system driven by linear motor has outstanding advantages in climbing ability, traction performance, vibration and noise. However, with the increase of traffic travel and other situations, how to ensure that the linear motor still has a large thrust and stability in the air gap condition is the key technical problem. In order to solve this problem, three Halbach permanent magnet array structures are proposed and applied to the magnetic barrier coupled primary permanent magnet unilateral excitation linear motor (MBCPPMUELM). The finite element software is used for simulation and analysis. By comparing the harmonic distribution of air gap magnetic field of the three Halbach permanent magnet array motors, a topology structure with high magnetic field modulation effect is obtained It provides a useful reference for the further design and analysis of this kind of motor.
\end{abstract}

Keywords: Halbach Distribution, Magnetic field, Linear Motor

\section{Introduction}

Urban rail transit has the outstanding advantages of pollution-free, large traffic volume, allweather and other outstanding advantages. Compared with the wheel rail transit system driven by traditional rotary motor, the linear motor rail transit system has good dynamic performance and climbing ability; the overall driving energy consumption is low; the vibration noise is low; The tunnel section is small and the construction cost is low; the safety, reliability, maintenance and repair quantity are low ${ }^{[1]}$. However, with the increase of urban rail transit distance, passenger capacity and other factors, ordinary permanent magnet linear motor can not meet the demand of long travel. It has important application value to design and develop a new linear motor drive system suitable for long stroke.

Halbach array is a new type of permanent magnet array, because its unique arrangement can enhance the magnetic density of one side of the magnetic field and weaken the magnetic density of the other side. As a result, the motor has a higher air gap flux density, reduces the harmonic distortion rate and harmonic loss of the air gap, and improves the torque ripple of the PMLSM motor ${ }^{[2]}$. After adopting the new Halbach array arrangement, the permanent magnet linear synchronous motor has better thrust density, meeting the

*Corresponding author: wangxpmail@163.com 
higher requirements of the contemporary society for the motor. There are many ways to arrange Halbach permanent magnet array in circular motor, but few in linear motor. In this paper, three kinds of Halbach permanent magnet arrays with different permutations are proposed in the case of the same amount of permanent magnet, and they are applied to the magnetic barrier coupled primary permanent magnet unilateral excitation linear motor (MBCPPMUELM) Maxwell software was used to simulate and analyse the magnetic line of force, magnetic field strength and air gap flux density of three Halbach permanent magnet arrangements in 18/20 MBCPPMUELM, and harmonic analysis was carried out. Figure 1 shows the structure of the 18/20 MBCPPMUELM.

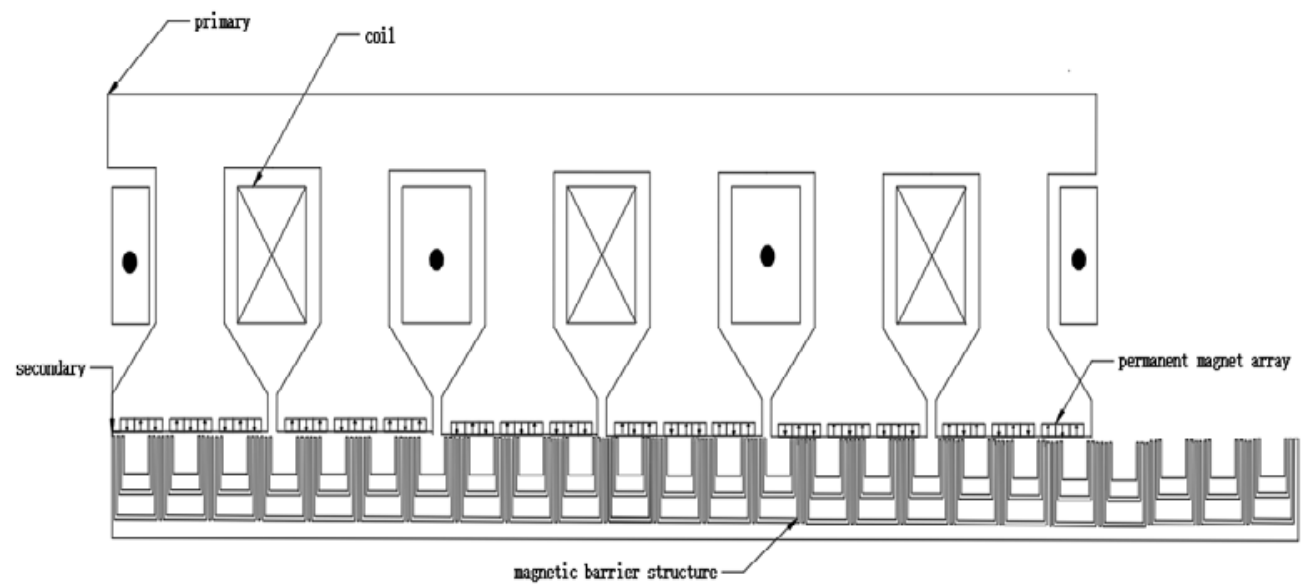

Fig.1. Structure of 18/20MBCPPMUELM.

\section{Topological Structure of Halbach Permanent Magnet Array}

Because Halbach permanent magnet array can effectively improve the performance of circular motor, but the application of Halbach permanent magnet array in linear motor is very few. Therefore, three kinds of Halbach permanent magnet array topologies with different arrangements are proposed and applied to 18/20 MBCPPMUELM.

\subsection{Semi Halbach permanent magnet array}

Each permanent magnet array is composed of three permanent magnets with equal width, height and thickness. The magnetizing direction of the middle permanent magnet is perpendicular to the motor motion direction and faces the air gap, which is used to generate the motor magnetic flux. There is the same angle between the magnetizing direction of the two permanent magnets and the magnetizing direction of the middle permanent magnet $(\theta$ in this paper is respectively taken as $30^{\circ}, 60^{\circ}, 90^{\circ}$ ). By adjusting this angle, the magnetic leakage at the edge of the permanent magnet is reduced, and the magnetic accumulation ability of the permanent magnet array is realized, in order to improve the motor performance The topology is shown in Figure 2.

\subsection{New Two-Stage Halbach Pole Array}

Each group of permanent magnet arrays has the same magnetizing law, and each array is composed of four permanent magnets with the same width, height and thickness. The angle between magnetizing direction and horizontal direction of each permanent magnet is $\theta$ ( $\theta$ 
$=30^{\circ}, 60^{\circ}$ ). This type of arrangement can have higher air gap flux density fundamental wave amplitude and smaller harmonic content in circular motor. The topology is shown in Figure 3.

\subsection{Continuous Magnetization of Permanent Magnets with Different Number of Blocks in Each Stage}

Halbach permanent magnet motor can be divided into 4 pieces of $45^{\circ}$ array of each pole, $60^{\circ}$ array of 3 pieces of each pole, $90^{\circ}$ array of 2 pieces of each pole and multiple array of each pole according to the quantity of magnetic materials and magnetizing direction of each pole. The more the number of permanent magnets under each pole, the better the effect of magnetic concentration, the better the sinusoidal air gap flux density, but the difficulty of installation will increase. Therefore, the first three permutation permanent magnet motors are widely used. The topology is shown in Figure 4.

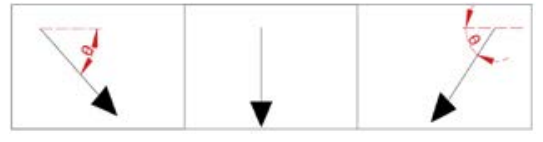

Fig. 2. Structure of Semi Halbach Permanent Magnet Array

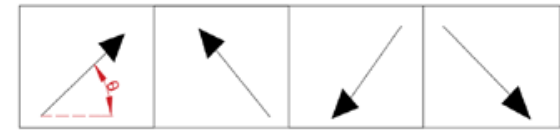

Fig. 3. Topological Structure of New TwoStage Halbach Pole Array

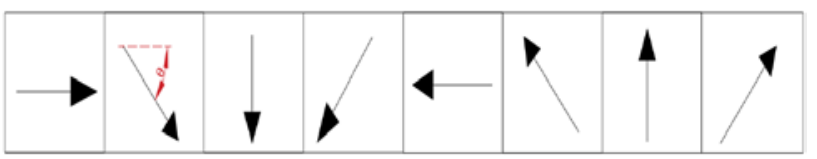

Fig. 4. Permanent Magnets with Different Magnets Per Stage

\section{Comparative Analysis of Motor Magnetic Field}

\subsection{Distribution of Magnetic Lines of Flux}

\subsubsection{Semi Halbach permanent magnet array}

18/20 MBCPPMUELM can modulate the magnetic field with a logarithm of 2 in the air gap. The magnetic field is analysed by finite element software.

Compared with the four kinds of magnetic lines of force, it is observed that when $\theta=$ $30^{\circ}$, the flux leakage of permanent magnet array and armature tooth edge is significantly reduced. This is because when $\theta=30^{\circ}$, the magnetomotive force component of permanent magnets on both sides in the positive direction of $\mathrm{x}$-axis decreases, and $\mathrm{Y}$ The results show that the magnetomotive force component in the negative axis direction increases, which reduces the magnetic flux between the permanent magnets and the armature teeth on both sides. To study the influence of different combinations of permanent magnet array structure on the magnetic field of the motor, it is actually the magnetic energy gathering force of the MMF component in the horizontal direction on the middle permanent magnetic flux in the array The best performance of semi Halbach permanent magnet array motor is achieved. Fig. 5 shows the distribution of magnetic force lines of semi Halbach permanent magnet array when $\theta=30^{\circ}$. 


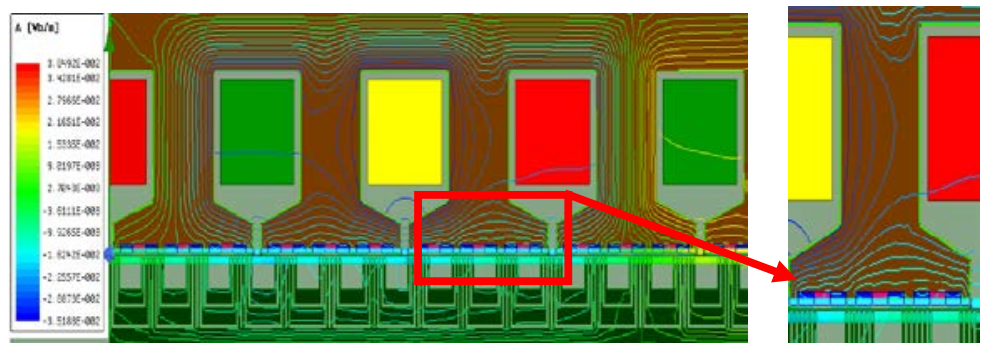

Fig. 5. Magnetic Line Distribution of Semi Halbach Permanent Magnet Array with $\theta=30^{\circ}$

\subsubsection{New Two-stage Halbach Pole Array}

The angle of magnetization is $30^{\circ}, 60^{\circ}$. When $\theta=60^{\circ}$, the leakage flux of permanent magnet array and armature tooth edge is almost the same as that of $\theta=30^{\circ}$, but the magnetic line of force in the primary yoke increases slightly. This is due to the fact that the magnetomotive force component of the permanent magnet in the positive direction of $\mathrm{x}$ axis is smaller than that when $\theta=30^{\circ}$ and the magnetic gathering ability between the permanent magnets is weakened. The increase of the MMF component in the negative direction of $\mathrm{Y}$ axis reduces the flux between the permanent magnet and the armature teeth, so the flux of $\theta=60^{\circ}$ is greater than that of $\theta=30^{\circ}$. Fig. 6 shows the distribution of magnetic force lines of the new two-stage Halbach pole array at $\theta=60^{\circ}$.

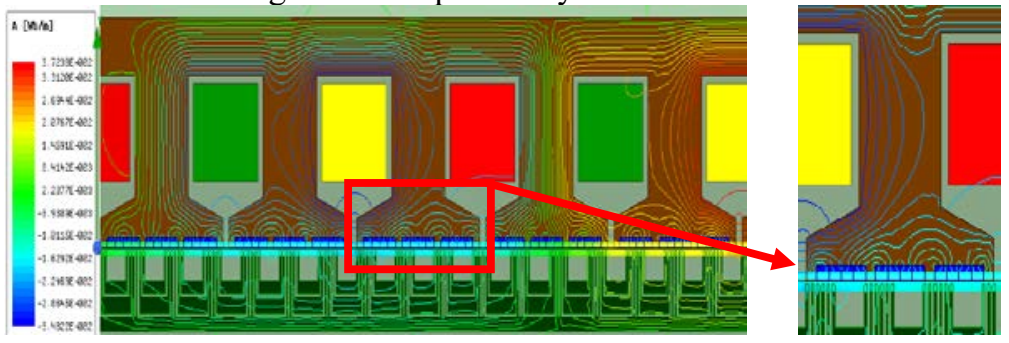

Fig. 6. Distribution of Magnetic Force Lines of New Two-Stage Halbach Pole Array at $\theta=60^{\circ}$

\subsubsection{Continuous Magnetization of Permanent Magnets with Different Number of Blocks Per Pole}

The simulation results show that when the magnetization direction $\theta=45^{\circ}$, there is a gap between the permanent magnet array and the armature tooth edge, which leads to the leakage of the two sides. However, the more the number of magnetic materials under each pole, the better the effect of magnetic agglomeration. Fig.7shows the distribution of magnetic lines of force when $\theta=45^{\circ}$ for four blocks per pole.

\subsection{Comparison of Magnetic Field Strength}

The density of Magnetic Lines of Flux can reflect the magnetic field strength of the motor. The distribution of magnetic field intensity is completely consistent with that of the magnetic line of force. The more dense the magnetic field lines are, the higher the magnetic field intensity is. The maximum magnetic field strength produced by the three Halbach permanent magnet arrays is shown in Table 1. 


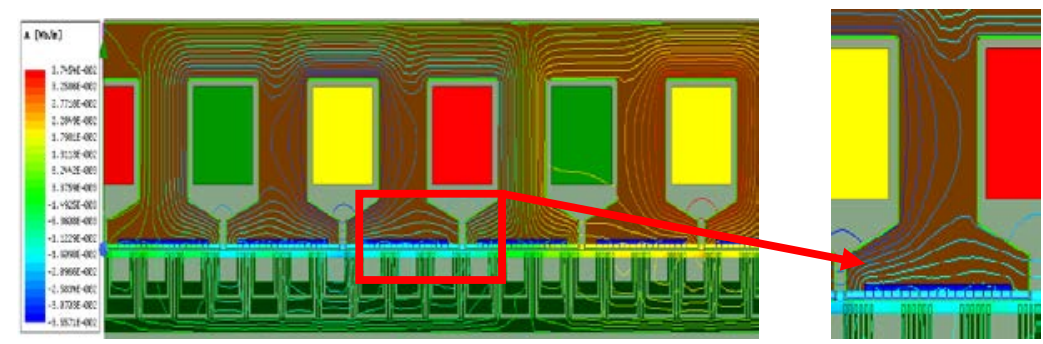

Fig. 7. The Magnetic Line of Force at $\theta=45^{\circ}$ Continuous Magnetization with Different Number of Blocks Per Pole

Table 1.Maximum magnetic field strength of three Halbach permanent magnet arrays

\begin{tabular}{|c|c|c|}
\hline \multirow{2}{*}{ Type } & Angle $\theta\left(^{\circ}\right)$ & $\begin{array}{c}\text { Magnetic induction intensity } \\
\mathrm{B}(\mathrm{T})\end{array}$ \\
\hline \multirow{3}{*}{\begin{tabular}{c} 
Semi Halbach permanent magnet $\begin{array}{c}\text { array } \\
\text { ary }\end{array}$ \\
\cline { 2 - 3 }
\end{tabular}} & $\theta=0^{\circ}$ & 2.029 \\
\cline { 2 - 3 } & $\theta=30^{\circ}$ & 2.455 \\
\hline \multirow{2}{*}{$\begin{array}{c}\text { New Two-stage Halbach Pole } \\
\text { Array }\end{array}$} & $\theta=60^{\circ}$ & 2.450 \\
\cline { 2 - 3 } $\begin{array}{c}\text { Continuous magnetization of } \\
\text { permanent magnets with different } \\
\text { numbers per pole }\end{array}$ & $\theta=90^{\circ}$ & 2.089 \\
\cline { 2 - 3 } & $\theta=60^{\circ}$ & 2.183 \\
\cline { 2 - 3 } & $\theta=60^{\circ}$ & 2.475 \\
\hline
\end{tabular}

It can be seen from table 1 that the magnetic field intensity produced by the half Halbach permanent magnet array $\left(\theta=30^{\circ}\right.$ and $\left.60^{\circ}\right)$, the Halbach permanent magnet array with two ends $\left(\theta=60^{\circ}\right)$, and the continuous magnetization of permanent magnets with different numbers per pole $\left(\theta=45^{\circ}\right.$ and $\left.90^{\circ}\right)$ is greater than the maximum magnetic field strength of $2.3 \mathrm{~T}^{[3]}$, It shows that the magnetic field strength can be improved by using the new Halbach permanent magnet, which is helpful to improve the working efficiency of the motor.

\section{Comparative Analysis of Air Gap Flux Density Harmonics of Motor}

The new Halbach permanent magnet array arrangement can provide larger air gap flux density and amplitude value for the motor, and the harmonic distortion rate of air gap flux density can be reduced, so that the linear motor has greater thrust and can meet the requirements of good operation in long stroke air gap working environment.

\subsection{Analytical Calculation of Air Gap Flux Density of Motor}

Ignoring the influence of high-order harmonics in the air gap magnetic field, only the DC component and the fundamental component in the air gap are considered. The value of noload air gap flux density B of MBCPPMUELM motor can be expressed by formula (1) ${ }^{[4]}$. 


$$
\begin{aligned}
& B=F_{p m(x)} \Lambda_{s}\left(x, x_{0}\right) \\
& =\left[F_{p m 0}+F_{p m 1} \cos \left(\mathrm{P}_{r} \frac{2 \pi}{L} x\right)\right]\left[\Lambda_{s 0}+\Lambda_{s 1} \cos \mathrm{P}_{c} \frac{2 \pi}{L}\left(x+x_{0}\right)\right] \\
& =F_{p m 0} \Lambda_{s 0}+F_{p m 0} \Lambda_{s 1} \cos \mathrm{P}_{c} \frac{2 \pi}{L}\left(x+x_{0}\right)+ \\
& \Lambda_{s 0} F_{p m 1} \cos \left(\mathrm{P}_{r} \frac{2 \pi}{L} x\right)+F_{p m 1} \cos \left(\mathrm{P}_{r} \frac{2 \pi}{L} x\right) \Lambda_{s 1} \cos \mathrm{P}_{c} \frac{2 \pi}{L}\left(x+x_{0}\right) \\
& =F_{p m 0} \Lambda_{s 0}+F_{p m 0} \Lambda_{s 1} \cos \mathrm{P}_{c} \frac{2 \pi}{L}\left(x+x_{0}\right) \\
& +\Lambda_{s 0} F_{p m 1} \cos \left(\mathrm{P}_{r} \frac{2 \pi}{L} x\right)+\frac{F_{p m 1} \Lambda_{s 1}}{2} \cos \frac{2 \pi}{L}\left(\mathrm{P}_{r}+\mathrm{P}_{c}\right)
\end{aligned}
$$

In formula (1): $F_{p m(x)}$ is permanent magnetomotive force; $F_{p m 0}$ is the amplitude of direct current component of magnetomotive force; $F_{p m l}$ is the amplitude of the first harmonic of magnetomotive force; $\Lambda_{s 0}$ is the amplitude of the direct current component of magnetic conductivity; $\Lambda_{s l}$ is the amplitude of the first harmonic of magnetic conductivity; $x_{0}$ is the difference between the magnetomotive force and the initial position of magnetic conductivity, that is the secondary initial position; $P_{c}$ is the number of teeth of the primary armature of the motor; $P_{c}$ is the number of secondary magnetic barrier poles;

The first and third terms are the direct current component produced by the permanent magnet in the air gap magnetic field and the fundamental wave component generated by the permanent magnet in the air gap magnetic field respectively. Both of these two terms do not interact with the secondary structure, so the back emf will not be generated in the winding with the movement of the secondary position;

The second term is that the secondary modulation teeth produce tooth harmonics in the breath;

The fourth and fifth terms are the air gap flux density harmonics generated by primary and secondary mutual modulation, respectively;

In the primary structure of the magnetic barrier coupled primary permanent magnet unilateral excitation linear motor, the modulation relationship between the number of permanent magnet pole pairs and air gap fundamental magnetic field pole pairs and the number of secondary magnetic barrier modulation teeth is ${ }^{[5]}$ :

$$
P_{c}=P_{p m}+P_{\text {air }}
$$

$P_{p m}$ is the permanent magnet pole number, $P_{\text {air }}$ is the air gap fundamental wave magnetic field pole number.

$$
\begin{aligned}
& P_{\mathrm{e}}=\left|P_{p m}-P_{c}\right| \\
& G_{r}=\frac{P_{c}}{P_{\text {air }}}=\frac{v_{e}}{v}
\end{aligned}
$$

$G_{r}$ is the modulation ratio of the motor; $v_{e}$ is the operating speed of the effective magnetic field; $v$ is the secondary mechanical speed; $P_{e}$ is the winding and logarithm;

The operation direction of the effective harmonic flux is the same as the primary operation direction of the motor. The traditional motor is the modulation motor with the modulation ratio $G_{r}=1$. The effective pole pairs are the permanent magnet pole pairs, and the magnetic field running speed is consistent with the mechanical speed. When the 
modulation ratio gr of the traditional motor is greater than 1 , the effective pole pairs are the pole pairs after the modulation of permanent magnet and secondary teeth, and the running speed of the magnetic field is $G_{r}$ times of the mechanical speed. At the same mechanical speed, the motor with the modulation ratio $G_{r}$ greater than 1 can obtain larger back EMF and thrust ${ }^{[6]}$, so the thrust density of MBCPPMUELM is higher than that of traditional permanent magnet motor.

\subsection{Comparative Analysis of Radial Flux Density Waveform of Motor}

The air gap flux density of three different Halbach permanent magnet arrays can be obtained by finite element simulation. Figure 8 shows the air gap flux density waveform of the semi Halbach permanent magnet array. The air gap flux density waveform of other structures can be obtained in the same way.

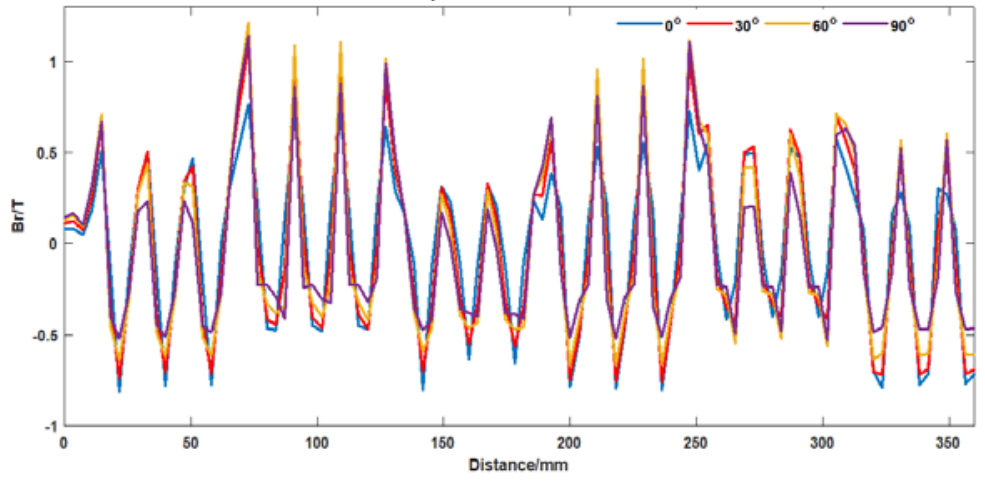

Fig. 8 Air Gap Flux Density Waveform of Semi Halbach Permanent Magnet Array

\subsection{Comparative analysis of harmonic amplitude}

It can be seen from Fig.9 that the three Halbach permanent magnet arrays are all used in the 18/20 MBCPPMUELM, and only the air gap harmonics generated by the permanent magnet alone are different. According to the principle of secondary magnetic field modulation, there are 2, 6, 12, 18 and 24 harmonics in the air gap magnetic field. The other parameters of the motor remain unchanged, only the permanent magnet magnetization direction is changed, and all the working amplitude values change with the change of magnetizing direction.

From the angle of air gap harmonic amplitude, as shown in Fig. 9(a), the harmonic order diagram of semi Halbach permanent magnet array is shown. When $\theta=30^{\circ}$, the maximum amplitude of 18th harmonic is $0.5116 \mathrm{~T}$. Fig.9(b) shows the harmonic order diagram of the new two-stage Halbach pole array. When $\theta=60^{\circ}$, the maximum amplitude of the 18th harmonic is 0.2964 T. Fig.9(c) shows the harmonic number diagram of permanent magnet with different number of blocks per pole. When $\theta=45^{\circ}$, the maximum amplitude of 18 th harmonic is $0.546 \mathrm{~T}$. 


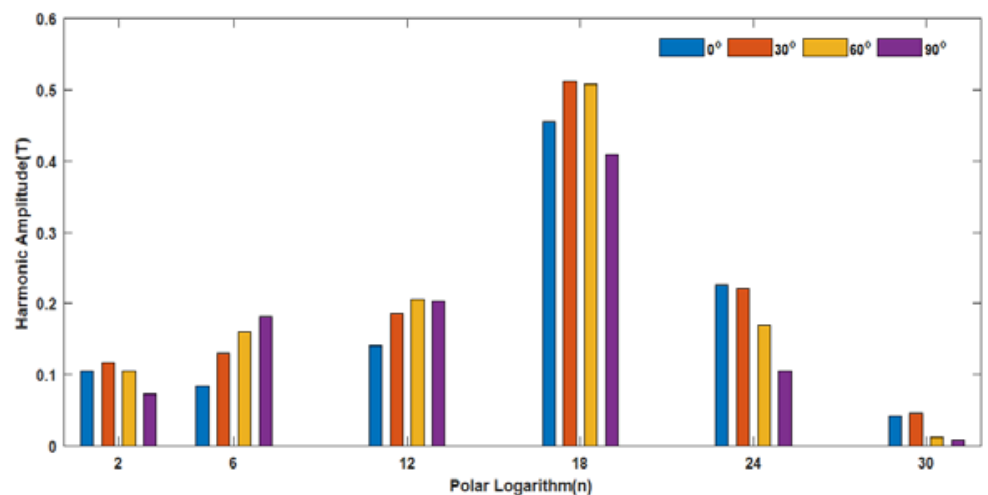

Fig. a. Harmonic Number of Semi Halbach Permanent Magnet Array

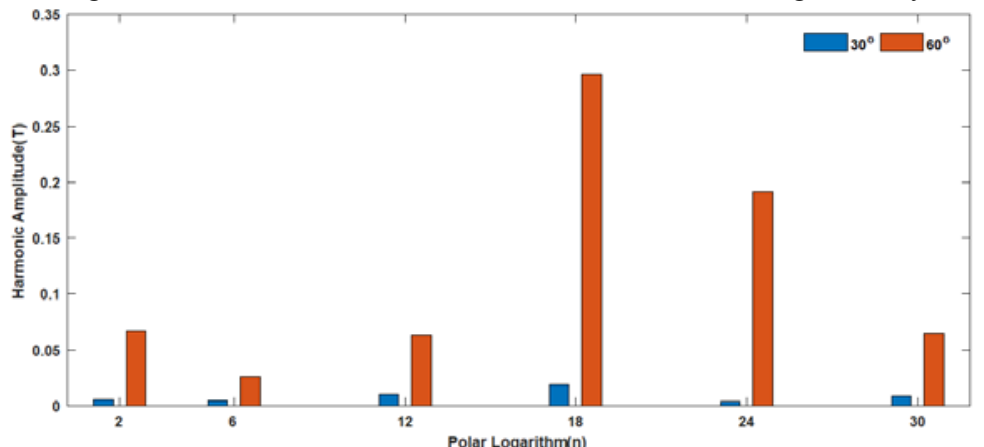

Fig. b. Harmonic Number of New Two-Stage Halbach Pole Array

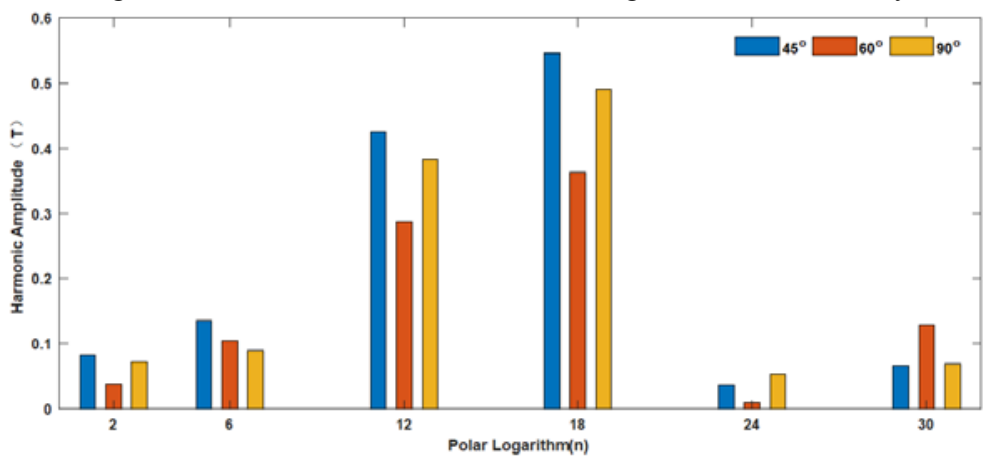

Fig. c. Harmonic Number of Permanent Magnets with Different Number of Blocks Per Pole

Fig.9. Harmonic Analysis of Four Halbach Permanent Magnet Arrays

\section{Summary}

At present, rail transit plays an increasingly important role in social development. Linear drive motor is an important component to ensure the safe and reliable operation of the system. In this paper, three different types of Halbach permanent magnet array structures are proposed on 18/20 MBCPPMUELM, which is of great significance to solve the problems of thrust reduction and power shortage of motor in long stroke and air gap working environment. The magnetic flux leakage of the three structures is analysed by finite element simulation software. The simulation results show that there are great differences in the harmonics of magnetic field, air gap flux density and air gap flux density. Through comparative analysis, it can be seen that the air gap flux density and harmonic 
amplitude of the three structures with $\theta=45^{\circ}$ and 4 pieces of each pole in semi Halbach permanent magnet array with continuous magnetization are larger Linear motor generates more thrust in the air gap, which is helpful to further improve the electromagnetic performance of the motor such as thrust density, positioning force and pulsation.

\section{Acknowledgments}

This project is financially supported by the National Natural Science Fund(51777127), Liaoning Natural Science Foundation (2020-MS-240),Innovative talents in Liaoning (Special motor and its control), Shenyang young and middle-aged scientific and technological innovation talents(RC200192),Liaoning Provincial Department of education scientific research project (JL-1907) and Liaoning million Talents Project .Thanking for the valuable suggestions from comrades.

\section{References}

1. Zhao Qing feng, Cheng Xiaomin. Application of Linear Motor in Urban Rail Transit System .J. Modern Urban Rail Transit, 2017, (10): 54-57

2. Xu Yanliang, Yao Fu'an, Fang Jiancheng. Halbach Magnet Structure Motor and Its Comparison with Conventional Magnet Structure Motor (II) -- Comparative Study on Magnet Structure and Magnetic Core Motor .J. Acta Electrotechnica Sinica, 2004, 19 (6): 58-62

3. Ji Yanwei. Research on Topology of Primary Permanent Magnet Linear Motor with Magnetic Barrier Coupling .D. Shenyang Institute of Engineering, (2020)

4. Zhu Jian. Design and Analysis of High Power Factor Bilateral Linear Vernier Permanent Magnet Motor .D. Jiangsu University,(2017)

5. Liu Yue. Research on Double Stator Multi Degree of Freedom Permanent Magnet Synchronous Motor .D. Harbin Institute of Technology, (2015)

6. Bian Fangfang. Research on Magnetic Field Modulation Mechanism and Topology of Primary Permanent Magnet Linear Motor .D. Jiangsu University, (2019) 